University of Nebraska - Lincoln DigitalCommons@University of Nebraska - Lincoln

CSE Conference and Workshop Papers

Computer Science and Engineering, Department of

2013

\title{
Field Study Identifying Barriers and Delays in Data- to-Decision with Small Unmanned Aerial Systems
}

Brittany A. Duncan

University of Nebraska-Lincoln, bduncan@unl.edu

Robin R. Murphy

Texas Aઐ'M University,murphy@cse.tamu.edu

Follow this and additional works at: http://digitalcommons.unl.edu/cseconfwork

Duncan, Brittany A. and Murphy, Robin R., "Field Study Identifying Barriers and Delays in Data-to-Decision with Small Unmanned Aerial Systems" (2013). CSE Conference and Workshop Papers. 241.

http://digitalcommons.unl.edu/cseconfwork/241

This Article is brought to you for free and open access by the Computer Science and Engineering, Department of at DigitalCommons@University of Nebraska - Lincoln. It has been accepted for inclusion in CSE Conference and Workshop Papers by an authorized administrator of

DigitalCommons@University of Nebraska - Lincoln. 


\title{
Field Study Identifying Barriers and Delays in Data-to-Decision with Small Unmanned Aerial Systems
}

\author{
Brittany A. Duncan and Robin R. Murphy \\ Department of Computer Science and Engineering \\ Texas A\&M University \\ College Station, TX 77843 \\ Email: \{bduncan, murphy\}@cse.tamu.edu
}

\begin{abstract}
This paper reports on lessons learned in rapidly getting data from a small tactical unmanned aerial system (sUAS) to an incident commander during a 2012 high fidelity hazardous materials exercise. In order to capture the Public Safety data-todecision path, observational data was collected on three flights of an AirRobot 100B sUAS, used extensively by the US Army, with HazMat specialists as part of a chemical train derailment

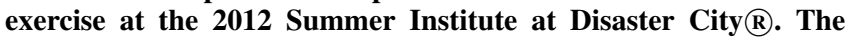
Summer Institute found that (i) the data path requires an average of 4 steps to go from the field to the incident commander, (ii) there is no standard data format which reduces the value of the data nor agreed upon paths for submission which leads to "broken" paths, (iii) redundant data-to-decision paths are essential in order to ensure information flow, and (iv) the average time from when the data was seen by the sUAS to its arrival at incident command was 27.8 minutes. The observations also led to three recommendations for companies producing devices: (i) sUAS should have a reliable capability to record to USB flash drive; (ii) all video and photographic imagery should have the relevant GPS and heading information embedded in the data; and (iii) systems should have the ability to provide cellular and wireless transmission capabilities (including web browsers and email) as responders may not have access to public phone Wi-Fi and internal Ethernet networks. The analysis also suggests that current measures of quality of service $(\mathrm{QoS})$ focus only on deviceto-device transfer rates, not the when the decision maker sees the data and if it is in a form to act upon.
\end{abstract}

\section{INTRODUCTION}

The Public Safety sector is exploring adoption of sUAS developed for the US Department of Defense. However, Public Safety agencies may have different informatics needs and communications may be limited. Thus, sUAS that work within the homogeneous operations and communications environment for the military may not fit Public Safety operations and communications constraints. This paper reports on lessons learned in getting data from a small tactical unmanned aerial system (sUAS) to an incident commander during a 2012 high fidelity hazardous materials exercise, i.e., the data-to-decision (D2D) process. The sUAS exercise was directed by Hazmat subject matter experts (SMEs) from fire rescue, environmental protection, and homeland security at the chemical train

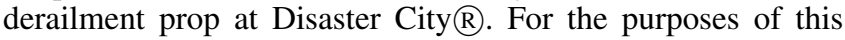
paper, the D2D is measured in terms of data transformations, which is the number of times a human has to intervene in the transmission process through copying data to new media or electronically manipulating the data, and the time delays between collection and delivery to a decision maker.

The paper contributes to the research and development of sUAS for Public Safety. Understanding the barriers in D2D process contributes to the theory of robotics and humanrobot interaction. It also provides manufacturers with insights in how to make their robot systems more attractive to the Public Safety sector. Section II introduces the related work, including both military D2D and Public Safety applications. The Summer Institute Field Study will be described in Section III. Section IV will discuss the different D2D paths that were attempted, whether successful or unsuccessful. The specific data transformations will be discussed by flight in Section V. Sources of time delay will be described in Section VI. The major findings from this field study will be presented in Section VII, with recommendations for robot manufacturers presented in Section VIII. Section IX offers the conclusion of this paper.

\section{RELATED WORK}

D2D has been an active area of research in the era of "big data", but not much is understood about the applications for Public Safety, rather than military, applications. Military D2D systems for unmanned systems provide a model of the Quality of Information (QoI), Value of Information (VoI), and Quality of Service (QoS), with the QoS measured with a focus on time delay in transferring data.

\section{A. Military Data-to-Decision with Unmanned Systems}

Bisdikian, et al. [1] discusses the development of an application-agnostic Quality of Information model to enable the sharing of sensor-originated information in military applications. The execution of this framework is beyond the scope of this paper, but the ideas discussed are extremely relevant. QoI is split into three properties: QoI, VoI, and QoS. QoI is about the judgment of the utility of the information in a stream, while the VoI is based on the information in the context of the receiver, and the QoS is based on the properties of the channels between the sender and receiver. VoI can be used to determine whether a particular source is right for the information sought, e.g., a ground vehicle is not valuable for an overview of a situation even though it has a high QoI. QoS is important when considering the data paths and whether information is arriving to Command in a way that it will be seen, e.g., an 
SD card sent to a command center without a card reader is unusable.

Blasch, Russell, and Seetharaman [2] examined data management to aid with D2D and showed how to process unstructured data, while leaving the QoI judgments to future work. In [2], there is a focus on the efficient exchange of data to support the D2D process. Data exchange incorporates challenging issues such as dissemination, quality, and timeliness, which is the focus of the rest of this paper.

\section{B. Public Safety Applications}

Public Safety applications may have a different set of priorities than military applications. For example, [3] reported that the highest priorities for a sUAS for wildland fire fighting were: the suitability of the sUAS for the task, the suitability for the environmental conditions, the distance the sUAS can fly, and the delivery of the data. The delivery of the data was defined as "who can get the data, how directly, and in what file formats ... and meta-data such as GPS location." This shows that QoI, VoI, and QoS are essential to adoption.

The Value of Information from a sUAS has been discussed in Murphy, et al. [4]. Two problems encountered at the Summer Institute 2012 as common complaints and concerns of emergency responders. These were: (i) lack of good optical acuity to read labels and placards and (ii) inability to review photographs in flight. Additionally, one of the common complaints described by Murphy was a lack of infrared payloads, of which one was used in this exercise exactly as she described it would be. The concern expressed is still valid because the FLIR payloads that have been used do not have the capability to record their own video nor to take pictures. Finally, Murphy noted that responders would like to have each image stamped with GPS location and orientation in order to aid integration into a map and to allow the vehicle to return to a point of interest.

\section{SUMMER INSTITUTE FIELd STUDY}

The Summer Institute 2012 Field Study at Disaster Cityßstaged a high fidelity reenactment of chemical train derailment. A simulated hazardous materials train derailment, shown in Figure 1, was used. Duplicating the insertion of sUAS at Hurricane Katrina [5], the robot operators presented an AirRobot 100B commonly used by the US Army to the incident commander. The AirRobot 100B is a quadrotor and representative of the typical interfaces and data acquisition capabilities of small rotorcraft. The team was tasked to provide the incident commander with a scene overview, chemical identification from placards and labels on the tanker cars, leak detection, and observation of tank fill levels using FLIR. The UAV team was made up of three to four members depending on the flight: a Pilot, a Safety Observer, and one to two Mission Specialists who were responders with hazardous materials expertise. A more thorough description of these roles can be found in [6]. Following the concept of operations in [5], the pilot was responsible for flying the sUAS under the direction of the mission specialist, who as the expert focused on accomplishing the objectives set by the incident commander. The sUAS team were required to leave the base of operations, enter the "warm zone," and maintain a stand-off

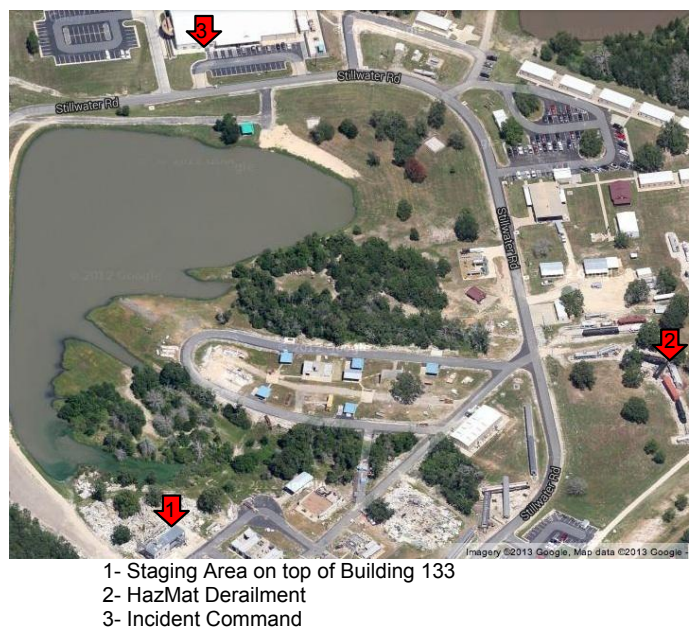

Fig. 1: Figure depicting operator placement, train derailment, and incident command.

distance of $305 \mathrm{~m}(1000 \mathrm{ft})$. Due to line-of-sight limitations on the sUAVs, the team chose to set up on top of a building just outside of the "hot zone". In order to accomplish the mission objectives, three flights were conducted over the train wreck. A fourth flight was conducted, but was unrelated to these objectives and the train derailment.

Data from the sUAS and meta-data on the D2D process was collected and archived using the RESPOND-R test instrument. The data sources were first person video from the pilot, flight $\log s$ from the UAV team, observational $\log s$ of the incident command activities from an ethnographer, and timestamps of the data and transfers from emails and pictures. Analysis was conducted by creating a timeline of the day, which focused on when photos were captured and the transformations they made along the way to incident command.

\section{SUMMARY OF DATA TRANSFORMATIONS}

The first measure of the D2D process is the number of data transformations. Data transformations followed seven different paths, shown in Figure 2, but only four were successful at completing the delivery to Command. Any time a human had to intervene in the data path, it is counted as a transformation (e.g., the thumb drive must be plugged into the base station and the record button must be pressed). Figure 2 shows the elapsed time as the length of a line, approximating the time or distance of transfer between the two boxes (walking a thumb drive to an access point takes less time than transferring over a low-bandwidth network).

There were three types of data that needed to be transmitted to Command: video from the robot, hi-resolution pictures from the robot, and lo-resolution pictures from the robot's perspective that were captured on the iPad. Each flight had the capability to take a video, but hi-definition pictures were based on the payload and were only available from the still picture camera, neither the FLIR nor the fixed-lens had the capability to take still pictures without the iPad.

Of the seven possible paths, only four were successful with 


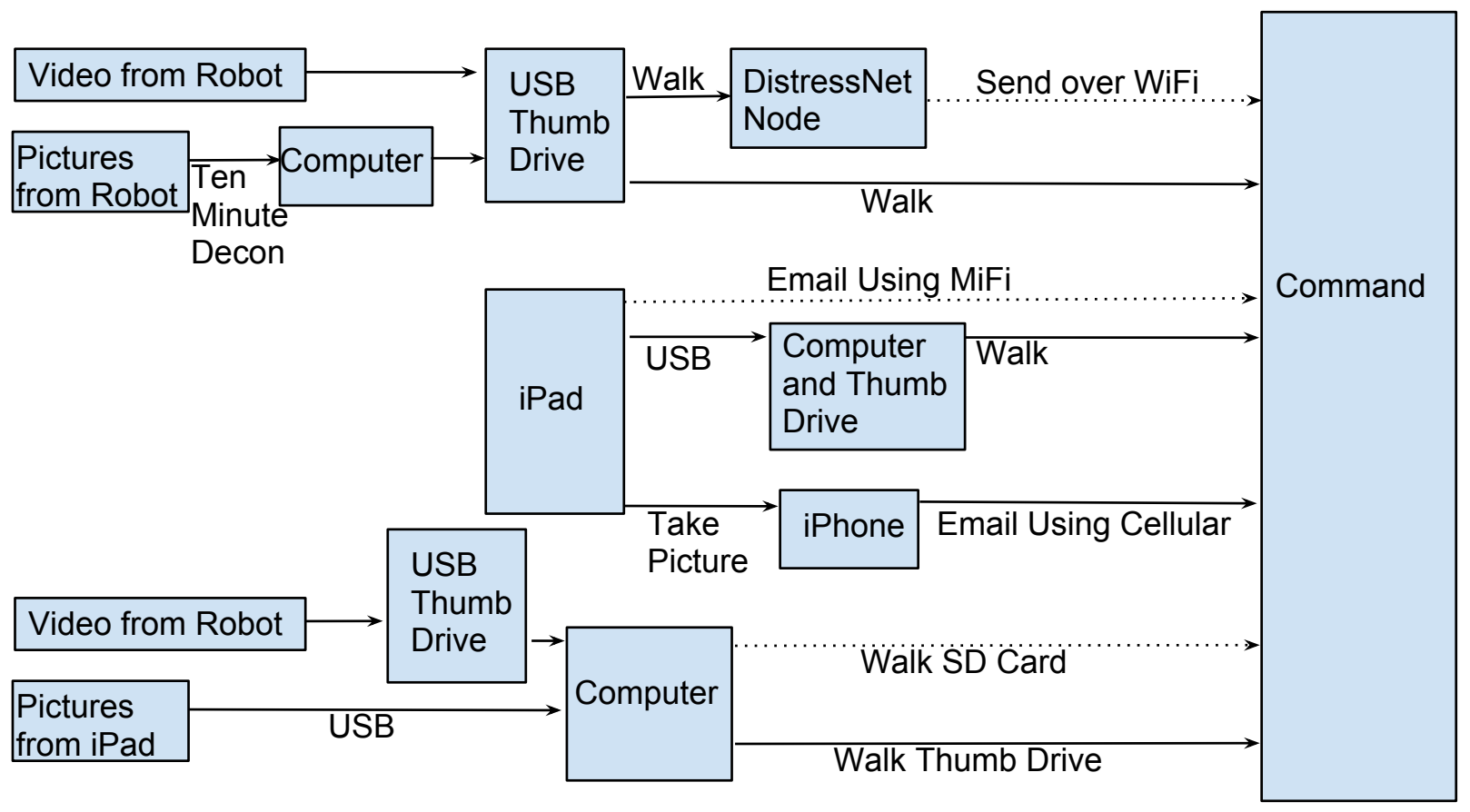

Fig. 2: Figure depicting data paths from operator to incident command.

the other three merely adding unnecessary steps to the path on the way to Command. Three of the successful paths were by "SneakerNet", which consisted of a person walking the data to Command in order to deliver a thumb drive. On the first flight, there were 3 steps to get the pictures on the thumb drive to Command, but the data took a two step detour to attempt wireless transmission which led to 5 steps for this flight. Flight 2 also took 3 steps for pictures one set of pictures and 2 steps for video via thumb drive to Command, with a 1 step detour to attempt transmission via $\mathrm{MiFi}$, leading to an average of 3.5 steps for this flight. Flight 4 took 3 steps for video and 2 steps for pictures to Command, with a 3 step detour to send an SD card to Command only to find out that they could not read an SD card and then to resend via thumb drive; the average for this flight was 5.5 steps. The final successful path was by taking a picture of the iPad interface with an iPhone and then emailing that picture to Command using the built in cellular network, which was a 2 step process. The average number of steps per completed path was 4 .

Each of the data paths presented here was used at least once throughout the flights, and some were used on multiple flights. The data paths will be discussed further in the next section, placed in the context of their flight, and the barriers will also be described.

\section{Data Transformation Paths}

The data transformation paths for each of the three sUAS flights merit additional discussion in order to better understand how the barriers to the data paths arose. The data paths for each flight are captured in Figures 3 to 5 and discussed below. The figures use the convention that a solid line with no arrowheads denotes a direct connection, while the addition of a single arrowhead shows a wired transfer. The dasheddot lines are representative of a wireless transfer, the dashed lines show a copy to new media, and the dotted lines show an unsuccessful transfer. The shapes are difficult to describe in text, but differentiate between a known expertise (e.g., command), data, and information or an interface.

\section{A. Flight 1}

The objective for Flight 1 was to provide the overview of the scene, and it took five steps for the transmission to reach Command. The flight plan was to go up to around $46 \mathrm{~m}$ (150 $\mathrm{ft}$ ) in the center of the incident and gain establishing shots for the incident commander. If there was still time remaining after the initial shots, individual pictures of the components of the incident were requested (e.g., crossed train cars) following a path straight over the incident from a lower altitude (dependent upon obstacles and line of sight considerations). Since the plan for this flight was based on prior field experience, mission specialist input was not as necessary and they were given a computer screen to watch to instruct the pilot to take pictures.

Due to the nature of the flight (needing high-level shots to establish the scene), the still camera payload was selected which could take 12 megapixel pictures to an internal SD card. Since the SD card is internal to the payload, the downside to these pictures is that they remained with the robot when it was in the decontamination area for 10 minutes post-flight. On this flight, 8 pictures were taken for a total of $41.8 \mathrm{MB}$. Other downsides to this payload were: that the timestamp data from 


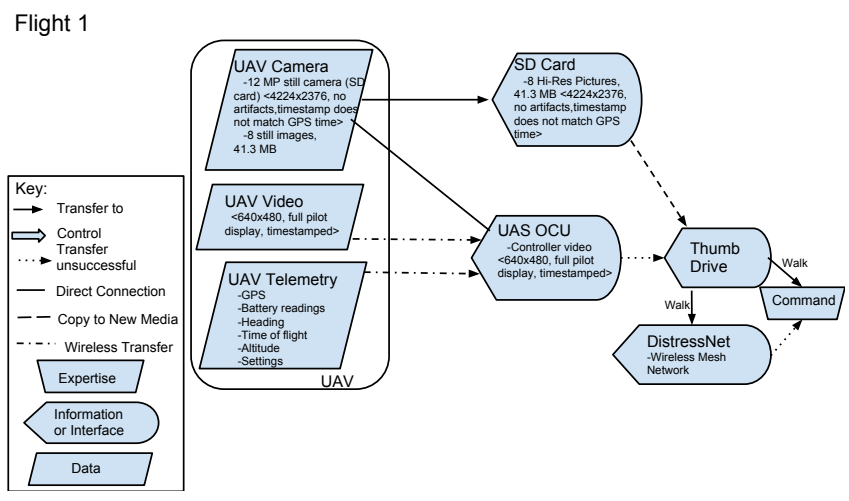

Fig. 3: The two attempted data paths and 5 steps of data transformation or transmission for Flight 1.

the pictures does not correspond to the GPS timestamp and there are no location artifacts with the pictures.

The video to Flight 1 should have provided the GPS, altitude, and heading data to supplement the pictures taken with the onboard camera. Unfortunately, this video was not saved due to operator error. The saving of the video from the operator control unit (OCU) is a three step process: a thumb drive must be inserted into the base station, the record button must be pressed to start the recording, and the stop button must be pressed to stop the recording before the OCU is powered off. The first two steps of this process were completed, but the final step was forgotten, so the recording was lost.

After both the flight and decontamination were complete, the pictures from the SD card were transferred to the thumb drive from the OCU in order to be plugged in to DistressNet [7] and transmitted to Command. As can be seen from Figure 3 , the use of DistressNet was unsuccessful. This is a great example of the lessons learned from the Summer Institute Exercise, because this network was designed for exactly such a purpose, but the researchers did not know what size files to expect, so it was unable to be used here. These researchers took these lessons back to their laboratory to continue work on DistressNet.

When the files were still not transferred after the next flight, the decision was made to send all of the data from both flights on a single thumb drive to Command via a person physically walking to the building where the incident commander was staged. This data path is simple and will be referred to as "SneakerNet". This path was chosen because Command needed data to make decisions, so it was necessary to send the pictures in the easiest and quickest way possible.

\section{B. Flight 2}

The objective of Flight 2 was to identify the chemicals being transported from placards and labels on the tanker cars and to detect any leaks. Additionally, the incident commander was looking for a confirmation that this was a radiological incident. Flight 2 added a Mission Specialist interface so that the specialized emergency responders could directly view the video and take their own pictures on the iPad, but this led to

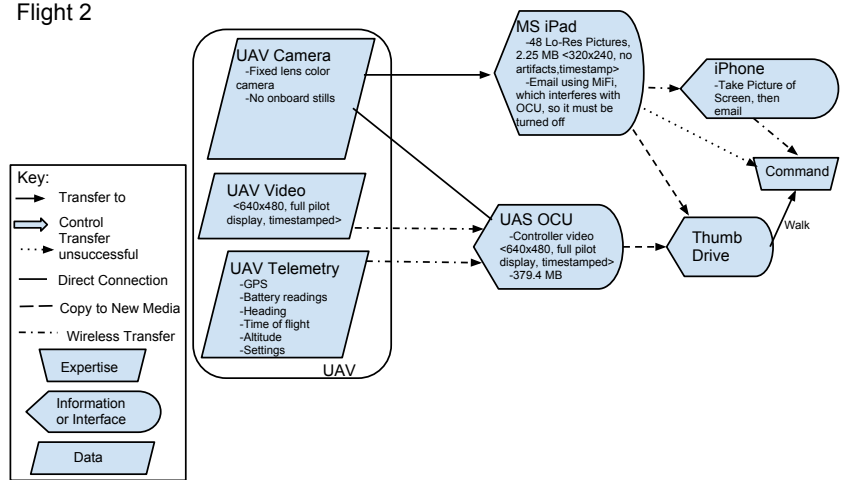

Fig. 4: The three attempted data paths and 6 steps of data transformation or transmission for Flight 2.

the team using two different data paths to Command (one was 2 steps and the other was 3-4 depending on the data type).

Due to the nature of the flight (needing better zoom to get shots of individual train car attributes), the fixed-lens camera payload was selected which could transmit video, but no pictures. The iPad became very important on this flight in order to take pictures, even though the ones captured were extremely lo-resolution $(320 \times 240)$. On this flight, 48 pictures were taken for a total of $2.25 \mathrm{MB}$. The timestamp data from the pictures corresponds to the iPad timestamp, which was set to the local time, but the location artifacts with the pictures referred to the location of the iPad, not the robot. The video to Flight 2 provided the GPS, altitude, and heading data to supplement the pictures taken with the iPad.

After the flight was complete, and during decontamination, the pictures from the iPad were transferred to the thumb drive from the OCU and two additional methods of transmission were attempted for the pictures, see Figure 4. The video from the flight was $379.4 \mathrm{MB}$, which is an excessive amount to try to send via email or file transfer, so this was only attempted by thumb drive. The iPad data was sent with the video, but in order to try to get visual confirmation of the radiological material to the commander as soon as possible, the single picture of the label was sent by taking a picture of the iPad with an iPhone and then emailing it via the cellular network. Finally, the iPad pictures were small enough that an attempt was made to send them via email through the use of a MiFi (personal cellular hotspot), but the use of this device interfered with the video feed from the robot, so its use was discontinued.

The data from both this flight and the previous were additionally transmitted on a single thumb drive to Command via a person physically walking to the building where the incident commander was staged. This data path is simple and will be referred to as "SneakerNet".

\section{Flight 4}

The objectives for Flight 4 were to confirm the location of the two leaks identified in Flight 2, where the leaking product might be flowing, and to try to get a confirmation of how full the leaking tanks were. Flight 4 also included the Mission 


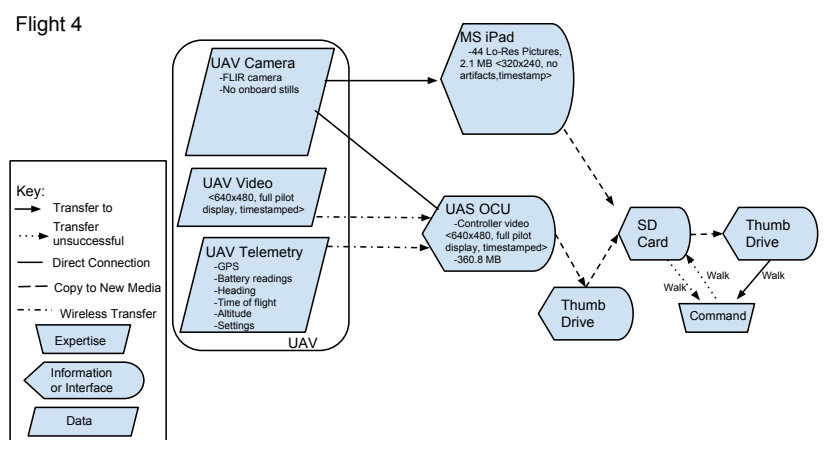

Fig. 5: The two attempted data paths and 6 steps of data transformation or transmission for Flight 1.

Specialist interface, and took up to 6 steps based on a flawed assumption that Command could read an SD card.

The FLIR camera payload was selected for this flight, and could transmit video, but no pictures. The iPad was also very important on this flight, even though the pictures were extremely lo-resolution (320x240). On this flight, 44 pictures were taken for a total of $2.1 \mathrm{MB}$. The timestamp data from the pictures corresponds to the iPad timestamp, which was set to the local time, but the location artifacts with the pictures referred to the location of the iPad, not the robot. The video to Flight 4 provided the GPS, altitude, and heading data to supplement the pictures taken with the iPad.

After the flight was complete, and during decontamination, the pictures from the iPad and the video from the OCU were both transferred to an SD card due to a shortage of spare thumb drives, see Figure 5. Due to the troubles from previous transmission attempts, "SneakerNet" was the only method attempted. When the SD card arrived at Command, it was determined that none of their computers had an SD card reader (a very interesting lapse in QoS), so it was sent back to be transferred to a thumb drive and was then resubmitted.

\section{ElAPSED TIME}

The second measure of the D2D process is the elapsed time between collection and delivery. As presented in the previous section, the average data path from the robot to Command involved 4 steps, it would be expected that such a high number of steps introduces latency into the delivery of data, but an average of 27.8 minutes seems excessive.

Flight 1 took an average of 43.6 minutes from the time the photo was captured until it was delivered to Command, with delays for decontamination of the robot and for a failed transmission via DistressNet. With the decision to use the hi-resolution still camera and its built-in SD card, this flight had a 10 minute delay for decontamination before the photos would be available, but it was determined that this delay was acceptable for the ability to zoom in on the pictures. The next area of delay was through trying to transmit via DistressNet; this delay was not one that required a team member to run, so we were able to accomplish Flight 2 while we were waiting. After the conclusion of Flight 2, it was determined that walking the data was the best option so the images from both flights were sent at the same time, but the walking added about 10 minutes of transmission time.

Flight 2 took an average of 25.5 minutes from the time the photo or video was captured until it was delivered to Command. With the additional capability of the iPad to take pictures locally, it was expected that this delay could be reduced significantly, but it still took time to transfer the pictures to a computer and to a thumb drive. One picture was prioritized and sent from an iPhone by taking a picture of the iPad picture, but even this picture was not sent until Flight 2 was complete and this picture alone gave no indication of where the materials would be located in the derailment because Command did not yet have any of the overview shots from Flight 1.

The Flight 4 data took an inconclusive amount of time due to the delays in delivery because of the misunderstanding about the ability of Command to use an SD card.

\section{FINDINGS}

The analysis of the D2D process provides four findings:

1) The data path requires an average of 4 steps to go from the field to the incident commander (e.g., data from operator control station over wireless, to a laptop with USB drive, which is then hand carried), versus a more desirable 1 step, such as sending data from the operator control station directly to the incident commander's laptop.

2) There is no standard data format which reduces the value of the data (e.g., imagery or video which is not geo-tagged) nor agreed upon paths for submission which leads to "broken" paths (e.g., the assumption that the incident commander's laptop can read a SD card when it cannot; use of low bandwidth mobile ad hoc networks that are not sufficient for transmitting imagery).

3) Redundant data-to-decision paths are essential in order to ensure information flow as the communications infrastructure evolves or encounters temporary failures.

4) The average time from when the data was seen by the sUAS to its arrival at incident command was 27.8 minutes. The observed data-to-decision process indicated that the sUAS team expended significant time and effort in data editing and data transfer, delaying additional flights and suggesting that a full time "data manager" be added to the team to free them from those duties.

\section{RECOMMENDATIONS}

Based on the analysis, ethnographic observations, and after action report from the responders, there are three recommendations for for improving the D2D process with sUAS.

1) sUAS should have a reliable capability to record video to a USB flash drive so that at worst case, data can be physically transported to the incident commander. Currently the standard is to record video from the vehicle in one of two ways: (i) record hi-definition video on the camera, or (ii) stream the video back 
to the ground station and record it there. The first approach runs into problems because this video is usually not synced with the telemetry data and will run into delays during decontamination of the robot. The second approach is problematic because it is generally video of a much lower quality. Both approaches are prone to user errors, but manufacturers should try to make the process as short and easy as possible. One approach might be to automatically start a recording on take-off and stop it on landing.

2) All video and photographic imagery should have the relevant GPS and heading information embedded in the data or as an overlay so that the command staff can correlate the data with their maps.

3) Systems should have the ability to provide cellular and wireless transmission capabilities (including web browsers and email) as responders may not have access to public phone Wi-Fi and internal networks. The reason that this recommendation is for the manufacturers is because of two issues encountered in this exercise: (i) the inability of an independently developed network to handle the bursty data of the sUAS, and (ii) the incompatibility of off-the-shelf solutions with the proprietary hardware of the robots. Developing these capabilities in-house will allow manufacturers to test hardware combinations and to choose solutions which will be compatible with their known data transmission requirements.

\section{CONCLUSION}

The exercise indicates that sUAS which fit military D2D processes are not compatible with Public Safety D2D expectations, but can be adapted with minor upgrades. The value of the information (VoI) from the sUAS was highly rated by the responders because the aerial vehicle provide new viewpoints that could be directed in real-time, but the quality of service (QoS) was low as evidenced by the large number of data transformations (avg. 4 steps) and elapsed time in getting data to the incident commander (avg. 28 minutes). The operational definition of D2D was able to highlight barriers in transferring data. This suggests that the network-oriented definition of QoS, as the quality of the medium being used to transport data, should be expanded to include the number of transformations. The analysis also poses the question of how to measure the relationship between VoI and QoS as it does not matter how quickly valuable information arrives at Command if they do not know it is there, or if they are unable to use the data effectively due to incompatible formats, poor displays, or lacking visualizations of the data; i.e., high QoS, high potential VoI, but unrealized in current command structure.

\section{ACKNOWLEDGMENTS}

This work was supported by a National Science Foundation Graduate Research Fellowship, NSF grant CNS-0923203 "MRI: Acquisition of Mobile, Distributed Instrumentation for Response Research (RESPOND-R)," and the Texas A\&M Engineering Experiment Station's Center for Emergency Informatics. The authors would like to thank Mr. Clint Arnett, Chief David Martin, and other members of the Texas A\&M Engineering Extension Service for designing and conducting the exercise.

\section{REFERENCES}

[1] Bisdikian, C., Kaplan, L.M., Srivastava, M.B., Thornley, D.J., Verma, D., and Young, R.I.: "Building principles for a quality of information specification for sensor information", in 'Building principles for a quality of information specification for sensor information' (2009, edn.), pp. 1370-1377.

[2] Blasch, E.P., Russell, S., and Seetharaman, G.: "Joint Data Management for MOVINT Data-to-Decision Making”. Proc. Information Fusion (FUSION) 2011.

[3] Murphy, R. R., "Potential Uses and Criteria for Unmanned Aerial Systems for Wildland Firefighting," in AUVSI Unmanned Systems North America, Denver, CO, 2010.

[4] Murphy, R.R., Peschel, J., Arnett, C., and Martin, D.: "Projected needs for robot-assisted chemical, biological, radiological, or nuclear (CBRN) incidents". Proc. Safety, Security, and Rescue Robotics (SSRR), 2012 IEEE International Symposium on, College Station, TX, USA 2012.

[5] Pratt, K. S., Murphy, R. R., Stover, S., and Griffin, C. "CONOPS and Autonomy Recommendations for VTOL SUASs Based on Hurricane Katrina Operations," Journal of Field Robotics, 2009, 26, (8), pp. 636650.

[6] Murphy, R. R., Pratt, K. S., and Burke, J. L. "Crew roles and operational protocols for rotary-wing micro-UAVs in close urban environments," In Proceedings of the Third ACM/IEEE International Conference on Human-Robot Interaction, pp. 73-80, 2008.

[7] George, S.M., Wei, Z., Chenji, H., Myounggyu, W., Yong Oh, L., Pazarloglou, A., Stoleru, R., and Barooah, P.: "DistressNet: a wireless ad hoc and sensor network architecture for situation management in disaster response", Communications Magazine, IEEE, 2010, 48, (3), pp. 128-136. 